The Type Eleven Secretion System

1 Title: Description of a widespread bacterial secretion system with chemically diverse substrates

2 Running title: The Type Eleven Secretion System

3 Byline: Alex S. Grossman ${ }^{\mathrm{a} \uparrow}$, Terra J. Mauer ${ }^{\mathrm{b} *}$ ฯ, Katrina T. Forest ${ }^{\mathrm{b}}$, and Heidi Goodrich-Blair ${ }^{\mathrm{a}, \mathrm{b}}$ \#

\title{
5 Affiliations:
}

6 aUniversity of Tennessee-Knoxville, Department of Microbiology, Knoxville, TN

7 bUniversity of Wisconsin-Madison, Department of Bacteriology, Madison, WI

$8 \quad$ \#Address correspondence to Heidi Goodrich-Blair, hgblair@utk.edu

9 *Present affiliation: Terra Mauer, Washtenaw Community College, Ann Arbor, MI

10 T These authors contributed equally to this work. 
The Type Eleven Secretion System interplay of microbial colonization factors, host surface conditions, and host immunological

\section{Abstract:}

(1)

In host-associated bacteria, surface and secreted proteins mediate acquisition of nutrients, interactions with host cells, and specificity of tissue-localization. In Gram-negative bacteria, the mechanism by which many proteins cross or become tethered to the outer membrane remains unclear. The domain of unknown function (DUF)560 occurs in outer membrane proteins throughout Proteobacteria and has been implicated in host-bacteria interactions and lipoprotein surface exposure. We used sequence similarity networking to reveal three subfamilies of DUF560 homologs. One subfamily includes those DUF560 proteins experimentally characterized to date: NilB, a host-range determinant of the nematode-mutualist Xenorhabdus nematophila, and the surface lipoprotein assembly modulators Slam1 and Slam2, which facilitate surface exposure of lipoproteins in Neisseria meningitidis $(1,2)$. We show that DUF560 proteins from a second subfamily facilitate secretion of soluble, non-lipidated proteins across the outer membrane. Using in silico analysis, we demonstrate that DUF560 gene complement correlates with bacterial environment at a macro level and host association at a species level. The DUF560 protein superfamily represents a newly characterized Gram-negative secretion system capable of lipoprotein surface exposure and soluble protein secretion with conserved roles in facilitating symbiosis. In light of these data, we propose that it be titled the type eleven secretion system

\section{(TXISS).}

\section{Importance:}

The microbial constituents of a host associated microbiome are decided by a complex responses. Filling such niches requires bacteria to encode an arsenal of surface and secreted

33 proteins to effectively interact with the host and co-occurring microbes. Bioinformatic

34 predictions of the localization and function of putative bacterial colonization factors are essential 
The Type Eleven Secretion System

35 for assessing the potential of bacteria to engage in pathogenic, mutualistic, or commensal

36 activities. This study uses publicly available genome sequence data, alongside experimental

37 results from representative gene products from Xenorhabdus nematophila, to demonstrate a role

38 for DUF560 family proteins in the secretion of bacterial effectors of host interactions. Our

39 research delineates a broadly distributed family of proteins and enables more accurate

40 predictions of the localization of colonization factors throughout Proteobacteria.

\section{Introduction:}

All plants and animals exist in association with bacterial symbionts that can contribute to

43 nutrition, protection, development, and reproduction. These symbionts express surface and

44 secreted proteins that facilitate host interactions through a variety of functions, including

45 acquisition of nutrients (e.g. TonB-dependent transporters in Gram-negative host-associated

46 bacteria $(3,4)$ ), interaction with host cells (e.g. Acinetobacter baumanii OmpA binding host

47 desmoplakin (5)), and specificity in host-range and tissue-localization (e.g. Vibrio fischeri pilin

48 variability mediating host specificity (6)).

Recently, a mechanism of lipoprotein surface tethering was identified in the human

50 pathogen Neisseria meningitidis and termed the Slam (Surface lipoprotein assembly modulator)

51 machinery $(1,2)$. Slam proteins containing the $\beta$-barrel domain DUF560 are required for surface

52 presentation of certain lipoproteins, including those that capture metal-carrying compounds used

53 by hosts to sequester nutrients from bacteria (1). Two N. meningitidis Slam proteins have been

54 characterized, each with distinct lipoprotein substrates. Slam activity also has been demonstrated

55 for DUF560 representatives from pathogens Pasteurella multocida, Moraxella catarrhalis, and

56 Haemophilus influenzae $(1,2)$. However, most Slam homologs have no bioinformatically

57 predicted substrate to date, and one study has found that $N$. meningitidis Slam1 can surface 
The Type Eleven Secretion System

58 expose unlipidated fHbp variants (7). Thus, the full functional potential of DUF560 proteins is

59 not yet known.

The DUF560 homolog NilB is a host-association and species-specificity factor in the

61 nematode symbiont Xenorhabdus nematophila, a proteobacterium in the family Morganellaceae

62 (8-10). A screen for X. nematophila mutants defective in colonizing Steinernema carpocapsae

63 intestines revealed the Nematode intestinal localization locus (Nil)(10, 11). The Nil locus

64 contains the genes $n i l B$ and $n i l C$, each of which is independently necessary for colonization of

65 nematodes. Biochemical and bioinformatic analyses have established that NilC is an outer-

66 membrane-associated lipoprotein and NilB is an outer-membrane $\beta$-barrel protein in the

67 DUF560 protein family with a $\sim 140$ amino acid periplasmic $\mathrm{N}$-terminal domain that contains

68 tetratricopeptide repeats (11-14).

To begin to understand the range of functions of DUF560 proteins, we assessed their

70 ecological distribution, genomic context, and relatedness. We experimentally examined the $X$.

71 nematophila DUF560 homolog HrpB, which is not predicted to interact with a lipoprotein

72 substrate. Finally, to better understand the potential role of DUF560 proteins in host-symbiont

73 interactions we analyzed DUF560 distribution among symbiotic Xenorhabdus. Our data

74 demonstrate that the activities of the DUF560 family extend beyond lipoprotein surface

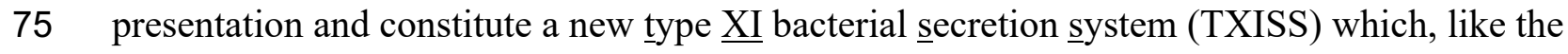

76 type II secretion system, is capable of acting on either membrane anchored or soluble proteins

$77 \quad(15)$ 
bioRxiv preprint doi: https://doi.org/10.1101/2020.01.20.912956; this version posted February 19, 2021. The copyright holder for this preprint (which was not certified by peer review) is the author/funder, who has granted bioRxiv a license to display the preprint in perpetuity. It is made available under aCC-BY-NC-ND 4.0 International license.

The Type Eleven Secretion System

\section{Results:}

\section{TXISS cluster according to environment}

Using homology to NilB or Slam proteins, previous work identified a wide distribution of

$$
\text { DUF560 proteins within mucosal associated bacteria }(2,10,11) \text {. To quantifiably delineate }
$$

subfamilies within the TXISS we generated a sequence similarity network (SSN) using the

$$
\text { Enzyme Function Initiative toolset (EFI) (16-18) and annotated it to highlight environmental }
$$

source or taxonomic grouping of the isolates containing DUF560 homologs (Fig 1; Supplemental

Table 1). In this network analysis, protein sequences with a high identity ( $40 \%$ or greater) were
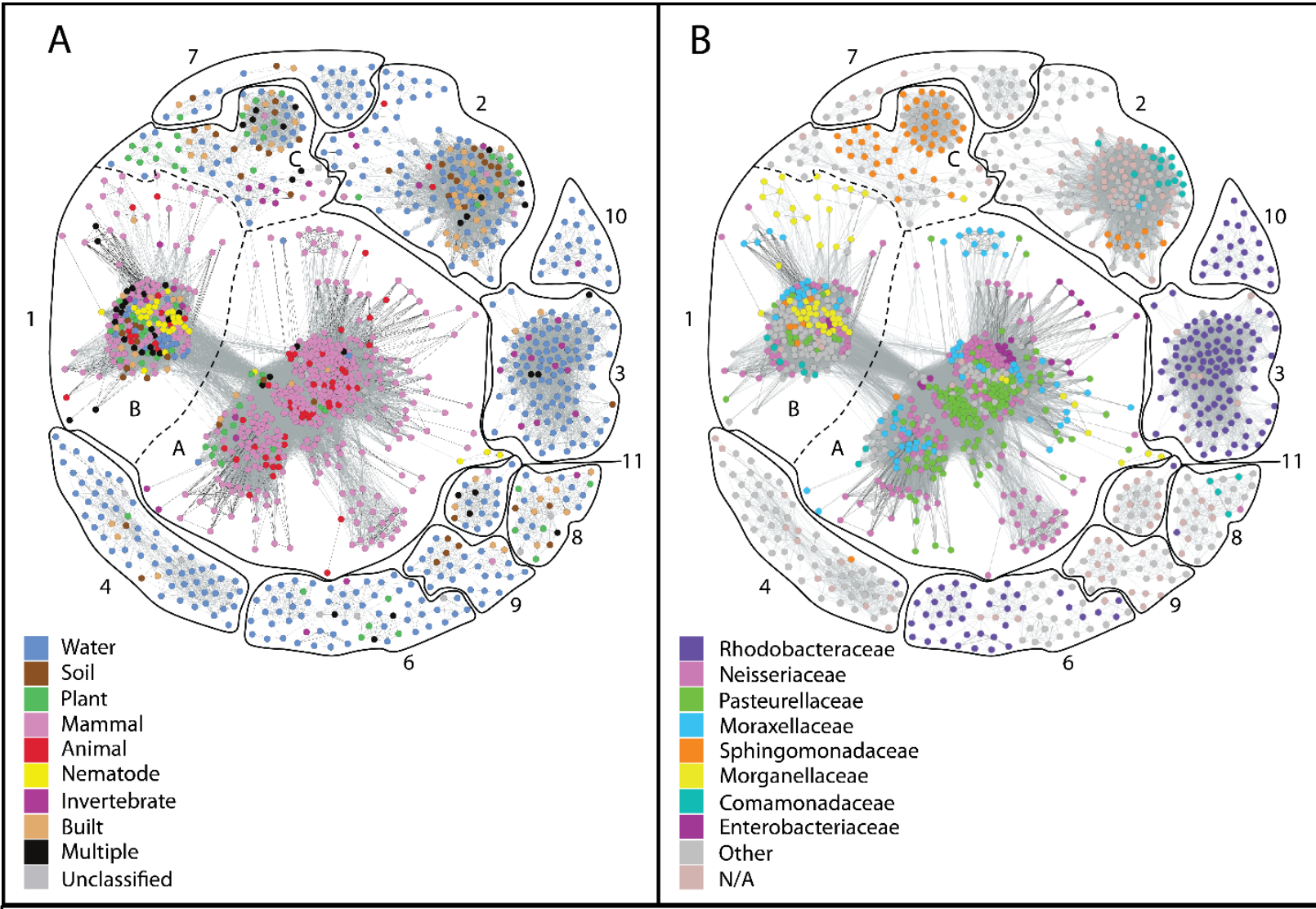

Figure 1. Sequence similarity network of all DUF560 proteins. SSN of DUF560 homologs generated by EFI-EST as accessed on 4.24 .19 (16-18). Edges were drawn using an alignment score of 38 , and any sequences which shared $\geq 40 \%$ identity were placed in a single node to allow the separation of clusters. Each node represents a group of highly similar sequences, with edge darkness demonstrating similarity, and the distance between nodes being determined via the Fruchterman-Reingold algorithm to optimize edge lengths (53). Each node was color coded to show the isolates' environmental origin(s) (A) and taxonomic class (B). 
The Type Eleven Secretion System

86 gathered into data points called nodes and connected together with edges based on sequence

87 similarity. Using all homologs in Interpro 73 and Uniprot 2019-02, we identified 10 major

88 clusters of TXISS proteins. Cluster 1 was chosen for in-depth analysis since it contained the

89 majority of nodes in the network (62.4\%) and could be visually divided into three subclusters

90 (1A, 1B, and 1C) using force directed node placement (Fig. 2). The remaining clusters displayed

91 a preponderance of water and soil associated organisms and contained no characterized proteins.

92 Consistent with our previous observations (11), the majority of cluster 1 nodes (75\%) comprise

93 sequences from various animal-associated isolates, while another $20 \%$ contain sequences from

94 marine, freshwater, soil, or built-environment isolates.

The division of nodes among the three subclusters more strongly reflected environmental

96 origin than bacterial taxonomy (Fig. 2A). Subcluster 1A almost exclusively comprises animal-

97 associated bacteria and contains all previously characterized TXISS, which separate according to

98 predicted substrate when analyzed with higher stringency (Supplemental Fig. 1). Subclusters 1B

99 and $1 \mathrm{C}$ have no previously characterized representatives. Subcluster 1B contains a mixture of

100 sequences from host-associated and free-living bacteria, while subcluster $1 \mathrm{C}$ contains sequences

101 largely from environmentally isolated Sphingomonadaceae. Subclusters 1A and 1B do not

102 correlate well with taxonomy of the isolates (Fig. 2B). For example, cluster 1 contains 81 nodes

103 with Moraxellaceae sequences. Of these, $79 \%$ are predominantly animal-associated and $12.3 \%$

104 are predominantly environmental isolates. The animal-associated isolates are enriched in

105 subcluster 1A and environmental-isolates are enriched in subcluster 1B. These data demonstrate

106 a correlation with lifestyle (e.g. free-living vs host associated) as opposed to taxonomy and

107 suggest that subclusters indicate divergent molecular functions. 
The Type Eleven Secretion System

\begin{tabular}{|c|c|c|}
\hline Environmental Classification & Taxonomic Classification (Family) & TbpBBD Association \\
\hline $\begin{array}{ll}\text { Water } & \text { Nematode } \\
\text { Soil } & \text { Invertebrate } \\
\text { Plant } & \text { Built } \\
\text { Mammal } & \text { Multiple } \\
\text { Animal } & \text { Unclassified }\end{array}$ & \begin{tabular}{lll|} 
Neisseriaceae & Alcaligenaceae \\
Pasteurellaceae & Enterobacteriaceae \\
Moraxellaceae & Other/Multiple \\
Sphingomonadaceae & N/A \\
Morganellaceae &
\end{tabular} & $\begin{array}{l}\text { TbpBBD sol co-occurrence } \\
\text { TbpBBD lip co-occurrence } \\
\text { TbpBBD sol and TbpBBD lip co-occurrence } \\
\text { TbpBBD without signal peptide } \\
\text { No detected TbpBBD co-occurrence }\end{array}$ \\
\hline Characterized Proteins & d/Slam1 Uncharacterized lipoprotein associated & HpuA associated/Slam2 $\bigcirc$ NilB \\
\hline \multicolumn{3}{|c|}{$\begin{array}{l}\text { Figure 2. Cluster } 1 \text { of the TXISS sequence similarity network (SSN) forms subclusters according to environment of isolation and } \\
\text { signal sequence of predicted cargo. All nodes from cluster } 1 \text { of the TXISS SSN were positioned using the Fruchterman-Reingold } \\
\text { algorithm. The resulting graph was annotated according to sequences A) environmental origin(s) or B) taxonomic class of the isolates, or C) } \\
\text { whether the node homolog(s) co-occur with a TbpBBD-domain encoding gene. Nodes containing experimentally characterized proteins are } \\
\text { highlighted using colored circles as indicated at the bottom of the figure. }\end{array}$} \\
\hline
\end{tabular}

111 facilitate surface exposure of lipoproteins, such as TbpB, LbpB, HpuA, and fHbp which are

112 frequently encoded nearby $(1,2)$. The lipid tail is not essential for Slam-dependent surface

113 exposure of a target $(1,7,19)$. This result prompted us to consider whether co-occurrence with

114 lipoproteins is a constant throughout cluster 1. We used the EFI Genome-Neighborhood Tool

$115(16,18,20)$ to assay the genomic context of each subcluster. This analysis corroborated previous

116 work demonstrating genomic association of DUF560 proteins with TonB, TonB-dependent

117 receptors and proteins that have a Pfam TbpB_B_D domain, which will be referred to hereafter

118 as $\operatorname{TbpBBD}(2,21)$. 


\section{The Type Eleven Secretion System}

121 gene structures to detect potential patterns. Using a combination of genome-neighborhood-

122 network data (18), Rapid ORF Description \& Evaluation Online (RODEO) data (22), and

123 manual annotation we analyzed all TbpBBD domain proteins co-inherited with sequences

124 present in cluster 1 (Supplemental Table 2). The majority of TbpBBD-bearing proteins

125 associated with subcluster 1A are predicted to be lipidated and have two TbpBBD domains

126 similar to $\mathrm{TbpB}$ in N. meningitidis (2), therefore they will be referred to as $\mathrm{TbpBBD}_{\text {lip. In }}$

127 contrast, the TbpBBD-bearing proteins associated with subcluster 1B are almost exclusively

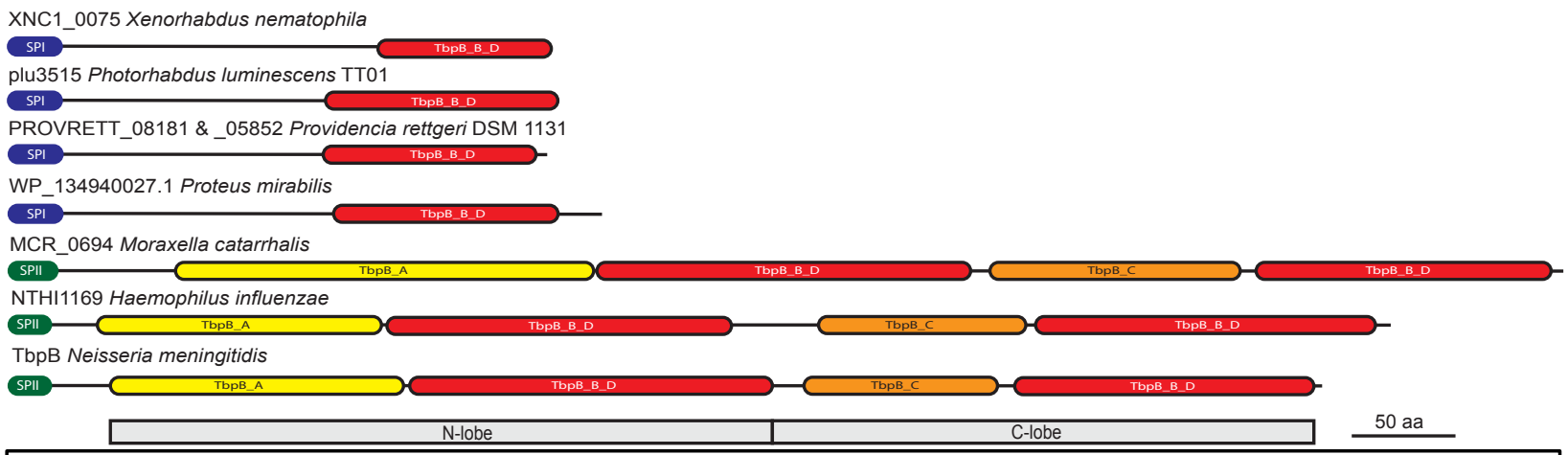

Figure 3. Examples of TbpBBD $_{\text {sol }}$ and TbpBBD lip domain architectures. The schematic diagram shows features found in select TbpBBD-domain containing proteins predicted to be exported by TXISS mechanisms. TXISS-1B associated proteins from X. nematophila, P. luminescens, P. rettgeri, P. mirabilis, and H. haemolyticus have signal-peptidase 1 (SPI) signal sequences, lack annotated "handle" domains, and have a single TbpBBD domain. TXISS-1A associated proteins from M. catarrhalis, H. influenzae, and N. meningitidis have signal-peptidase 2 (SPII) type signal sequences, N-lobe and C-lobe handle domains (TbpB_A and TbpB_C, respectively), and two TbpBBD domains. Predicted signal peptidase sites and domains are color-coded. predicted to be soluble proteins and only have a single TbpBBD domain similar to hemophilin in 
The Type Eleven Secretion System

133 between hemophilin and several $\operatorname{TbpBBD}_{\text {sol }}$ proteins $(24,25)$ (Supplemental Fig. 2). In light of

134 sequence and structural level similarities we hypothesized that subcluster 1B proteins transport

$135 \mathrm{TbpBBD}_{\text {sol }}$ proteins across the outer membrane via a mechanism analogous to subcluster

136 1A proteins transporting $\mathrm{TbpBBD}_{\text {lip }}$ substrates. Our prediction separates the TXISS into two

137 subfamilies, TXISS-1A which has members that flip TbpBBD lip $_{\text {substrates across the outer }}$

138 membrane and TXISS-1B which we predict has members that secrete $\mathrm{TbpBBD}$ sol substrates into

139 the extracellular milieu.

\section{TXISS-1B activity reconstructed in vivo}

To test our prediction that the TXISS-1B can secrete $\mathrm{TbpBD}_{\text {sol }}$ substrates, we

143 investigated the heme receptor protein (Hrp) locus of $X$. nematophila. This locus, which is

144 conserved across the Xenorhabdus genus, consists of genes predicted to encode TonB, a TonB-

145 dependent heme receptor named HrpA (XNC1_0073), the TXISS-1B homolog HrpB

146 (XNC1_0074), and its predicted TbpBBD sol $_{\text {substrate }} \operatorname{HrpC}(\mathrm{XNC1}$ _0075) (11), a homolog of

147 the heme-binding protein hemophilin (55\% similar 39\% identical) (23). Specifically, we sought

148 to test whether the TXISS-1B homolog HrpB mediates secretion of the putative heme-binding

149 protein HrpC. Using the expression vector pETDuet-1, HrpC-FLAG was expressed with and

150 without HrpB-FLAG and whole cell and supernatant fractions were separated and analyzed by

151 immunoblotting with anti-FLAG antibodies (26). We found that in the presence of HrpB-FLAG

152 the levels of HrpC-FLAG detected in the supernatant increased 9.9-fold at 1-h post-induction and

153 17.0-fold at 2.5-h post-induction (Fig. 4A). Whole cell lysates demonstrated equivalent

154 expression of HrpC-FLAG in both treatments (Fig. 4B). Coomassie staining of supernatants

155 show that expression of HrpB-FLAG did not induce cell lysis or non-specific protein secretion 
The Type Eleven Secretion System

(Fig. 4C, Supplemental Fig. 3). Consistent with the fact that in the absence of Slam1, several

strains of $E$. coli surface expose a fraction of the substrate fHbp $(27,28)$, some HrpC-FLAG

reached the supernatant in the absence of HrpB-FLAG.

The Hrp locus is conserved in many proteobacteria including Proteus mirabilis,

example, the $\mathrm{TbpBBD}_{\text {sol }}$ protein present in $N$. gonorrhoeae (NGO0554) is flanked by a gene

predicted to encode a TonB-dependent receptor (NGO0553) and a gene predicted to encode a

DUF560 family protein (NGO0555). Consistent with the predicted role of these $\mathrm{TbpBBD}_{\text {sol }}$

proteins in metal homeostasis, NGO0554 is repressed by iron, upregulated in response to

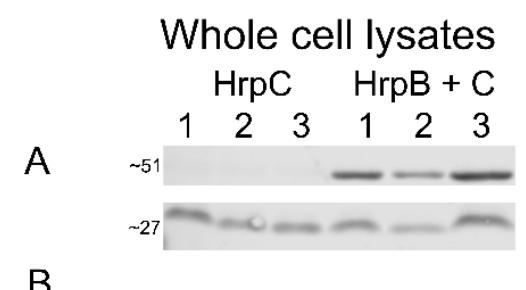

B
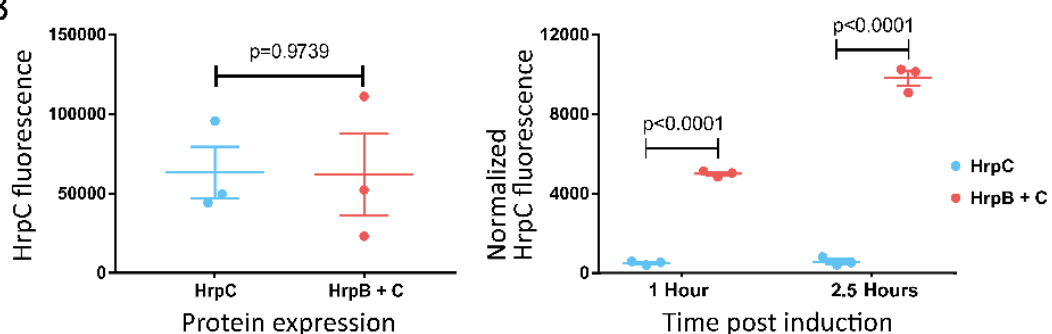

\section{Supernatants}

$$
\mathrm{HrpC} \quad \mathrm{HrpB}+\mathrm{C}
$$
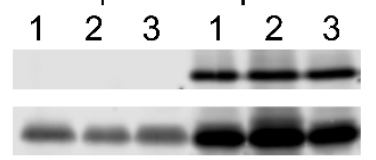

Time post induction

Figure 4. HrpB increases secretion of HrpC. A) Representative immunoblots of $2.5 \mathrm{~h}$ post-induction whole cell lysates and supernatant samples. HrpC-FLAG and HrpB-FLAG were distinguished by molecular weights ( $\sim 27$ and $\sim 51 \mathrm{kDa}$, respectively). B) Fluorescence intensity of HrpC levels was not significantly impacted by HrpB in cell lysates (left graph, unpaired t-test) but was in supernatants (right graph, Sidak's multiple comparisons test). C) Representative Coomassie stained SDS-PAGE gel of supernatant samples indicating HrpB causes neither cell lysis nor nonspecific protein export. A distinct band (in orange) was used as a loading control to normalize supernatant samples. The increase in supernatant $\mathrm{HrpC}$ is faintly visible at $\sim 27$ kilodaltons in the last three supernatant lanes. Relicates labeled 1-3. 
bioRxiv preprint doi: https://doi.org/10.1101/2020.01.20.912956; this version posted February 19, 2021. The copyright holder for this preprint (which was not certified by peer review) is the author/funder, who has granted bioRxiv a license to display the preprint in perpetuity. It is made available under aCC-BY-NC-ND 4.0 International license.

The Type Eleven Secretion System

\section{Host environment drives TXISS class}

Having established that DUF560 homologs represent a bona fide secretion system, we

173 next used the Xenorhabdus system to expand on our observation that the presence and type of

174 TXISS corresponds to bacterial environmental niche. Xenorhabdus are species-specific obligate

175 mutualists of Steinernema nematodes, and NilB is a known host range determinant. Therefore,

176 we considered host species as an environmental niche and bioinformatically examined whether

177 the complement of DUF560 genes in a microbe corresponds with host phylogeny (34-38). All 46

178 Xenorhabdus genomes on the Magnifying Genome (MaGe) platform (39) encode between one

179 and three TXISS, with five distinct homologs represented across the genus (one TXISS-1A and

180 four TXISS-1B) (40). Each unique combination of TXISS homologs was assigned one of six

181 classes (A-F) as depicted in Supplemental Data Fig. 4 and Supplemental Table 3. To visualize

A

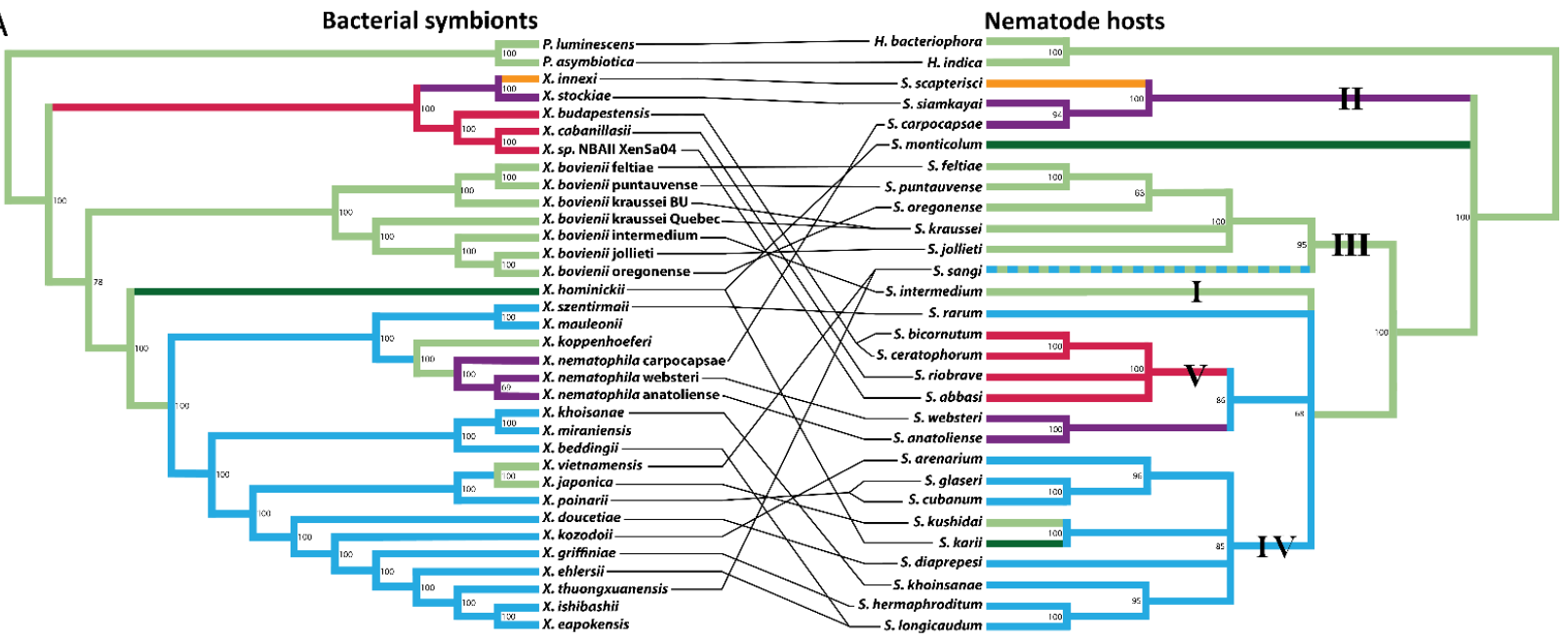

Class A- Hrp

Class B- Hrp + Hrp2

Class C- Hrp + HrpTn

Class D- Hrp + Cobalt utilization

Class E- Hrp + Cobalt utilization + Nil

Class F- Hrp + Nil

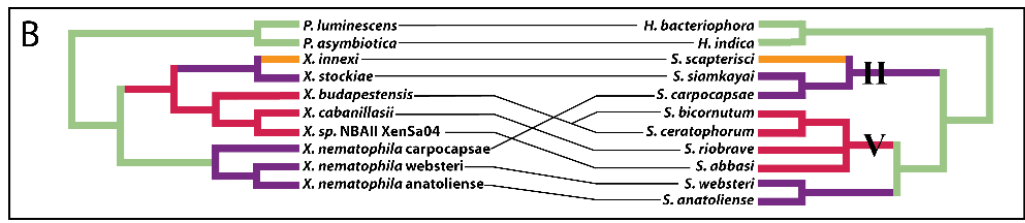

Figure 5: Cladograms of Xenorhabdus and Steinernema color coded according to Xenorhabdus DUF560 Class. Co-phylogeny of nematode species and their colonizing bacteria A) across the Steinernema genus or B) with a focus on specific clades. Numbers on branches indicate bootstrap support values. Bootstrap values below $60 \%$ were contracted. Lines connecting the phylogenies indicate mutualist pairs. Roman numerals highlight the 5 Steinernema clades described by Spridinov et al. 2004. Colored overlays indicate the DUF560 class of a given bacterium or a given nematode's symbiont. Class A (light green); class B (light blue); class C (dark green); class D (red); class E (orange); class F (purple). 
The Type Eleven Secretion System

182

183

184

185

186

187

correlations between TXISS class and host identity, we constructed Maximum Likelihood and Bayesian phylogenetic trees for Xenorhabdus and Steinernema. Xenorhabdus trees were generated with whole genome data while Steinernema trees used five loci as available (Supplemental Fig. 5; Supplemental Table 4). Aligning the Maximum likelihood phylogenies reveals that the TXISS complement of a Xenorhabdus species is more predictive of nematode host than phylogenetic position (Fig. 5).

This alignment of the phylogenetic placement of a given Steinernema host with the

TXISS complement of the symbiont provides insights into the nematode internal environment experienced by the symbiont. For example, most class B/C Xenorhabdus with two HrpB paralogs at the Hrp locus are symbionts of nematodes within the phylogenetic Clade IV, suggesting that these nematodes present a distinctive environment in which an additional Hrp locus is beneficial. Class D Xenorhabdus with an HrpB paralog encoded adjacent to genes predicted to encode a cobalt $\mathrm{ABC}$ transporter, are solely symbionts of Clade $\mathrm{V}$ nematodes. $X$. innexi and $X$. stockiae have seemingly diverged from this lineage through acquisition of a nilB homolog and switching to hosts within Clade II. Similarly, X. nematophila independently gained nilB and switched into a Clade II host (Fig. 5B). These acquisitions, alongside the varied genomic contexts of nilB/nilC pairs, are consistent with previous suggestions that the Nil locus was horizontally acquired among Xenorhabdus (14).

The TXISS NilB and the lipoprotein NilC are necessary for X. nematophila to colonize the Clade II nematode $S$. carpocapsae $(10,14)$. However, $X$. nematophila also colonizes two nematodes that are phylogenetically separate from Clade II, S. anatoliense and S. websteri (Fig. 5). Our hypothesis that TXISS are involved in host-environment adaptations leads to the prediction that $X$. nematophila will require the Nil locus to colonize these nematodes. To test this 
The Type Eleven Secretion System

A.

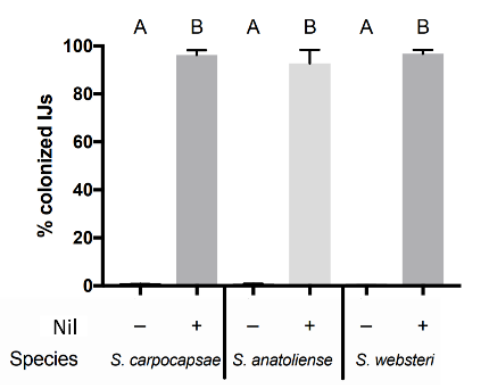

B.

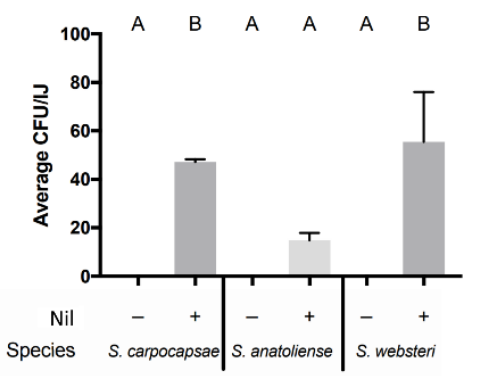

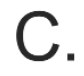

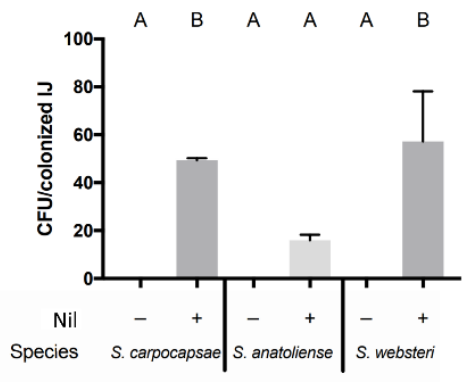

Figure 6. The Nil locus is necessary for colonization of $\boldsymbol{S}$. anatoliense, $\boldsymbol{S}$. carpocapsae, and $\boldsymbol{S}$. websteri infective juveniles. Bacteria free S. carpocapsae, S. anatoliense, and S. websteri were exposed to GFP expressing X. nematophila lacking or bearing the Nil locus. The resulting progeny infective juveniles (IJs) were monitored for colonization either by A) microscopy or by B) plating lysates for average CFU/IJ. C) The average CFU per colonized IJ combines both of these values to show bacterial load per organism. Treatments were analyzed via one-way ANOVA and Tukey's post-hoc test.

with our prediction, the $\Delta$ nil mutant was deficient in infective juvenile colonization in all three nematode species (Fig. 6), demonstrating that nil genes are necessary for infective juvenile colonization of $S$. anatoliense and $S$. websteri and supporting our hypothesis that TXISS promote adaptation to host environments.

\section{Discussion:}

216 cell membranes depending on the effector. Bacteria have evolved specialized secretion systems

217 for delivery of effectors that facilitate the host-associated lifestyle. For instance, the type II

218 secretion system can secrete hydrolyzing enzymes essential for commensals to grow, but it can

219 also secrete clinically important toxins such as Cholera toxin and Diphtheria toxin $(42,43)$.

220 Other secretion systems such as type III and type VI are specialized for the delivery of effectors 
The Type Eleven Secretion System

221 across both bacterial and host cell membranes. Knowledge of cargo protein identities and sorting

222 processes facilitates predictions from genomic information of bacterial secretome composition,

223 regulation, and localization. Despite the diverse secretion systems now recognized, there are

224 more to be identified based on the fact that some proteins predicted to be secreted lack known

225 secretion pathways (44). The type X secretory pathway was described in 2020 and shed light on

226 long standing mysteries surrounding the dependence on a muramidase for secretion of Typhoid

227 toxin across the cell wall (45). Secretion pathways are the tools with which bacteria interact with

228 their world, and as such each one discovered provides a slightly more complete picture of the

229 toolbox.

The DUF560 (domain of unknown function) family presence in animal-associated

231 bacteria was first recognized when it was noted that the host-colonization factor NilB has

232 homologs in N. meningitidis, M. catarrhalis, P. multocida, and H. influenzae (10). This

233 observation was strengthened by subsequent demonstration that Slam1 from human pathogenic

234 bacteria facilitates the surface presentation of host-metal acquisition proteins $(1,2,10)$. Enabled

235 by the availability of extensive genomic data from many environments and ever-improving

236 bioinformatic visualization tools, we have presented data demonstrating that the type XI

237 secretion system is a broadly distributed molecular vehicle for moving proteins across the Gram-

238 negative outer membrane. While DUF560 proteins were originally thought to represent a

239 mechanism for lipoprotein surface exposure (1), recent studies have expanded that functional

240 range to include peripheral membrane proteins (7) and now soluble secreted proteins,

241 establishing the function of DUF560 OM proteins in secretion of varied substrates. We predict

242 that further study will uncover a great diversity of cargo for the distinct classes of TXISS

243 revealed through our network analysis. Here we focused on one single cluster of a DUF560 
The Type Eleven Secretion System

244 sequence similarity network and highlighted two subfamilies of TXISS (TXISS-1A and 1B). The

245 remaining 9 clusters likely represent diverse subfamilies responsible for transporting as-yet-

246 unknown cargo. The lipoprotein cargo proteins for which structural data are known revealed a

247 common C-terminal 8-stranded $\beta$-barrel motif (2). Our discovery that HrpC is a cargo protein for

248 TXISS-1B HrpB strengthens the concept that this barrel is an important characteristic of TXISS

249 cargo; HrpC is a homolog of the H. haemolyticus hemophilin, the structure of which likewise

250 adopts a C-terminal 8-stranded $\beta$-barrel (23) (Supplemental Fig. 2). These data support the

251 concept that TXISS cargo have bifunctional structures in which the N-terminus is the host

252 effector domain while the C-terminus directs the protein for secretion. This framework will

253 facilitate identification of as-yet-unknown TXISS cargo among the genes that co-occur with

254 TXISS OMPs. Further, our analyses suggest that sequence similarity network clusters have

255 predictive power for other characteristics of TXISS cargo, including whether they are surface-

256 attached or secreted. The network-enabled classification presented here will facilitate the

257 investigation of both TXISS OMPs and their cargo in diverse bacteria.

258 Our work enables categorization of TXISS within Neisseria species that are found in

259 many animal hosts, including the human pathogens $N$. meningitidis and N. gonorrhoeae (46).

260 Neisseria strains can encode up to 6 TXISS paralogs. N. meningitidis MC58 has two functionally

261 characterized TXISS: Slam1 and Slam2. Our network analysis indicates N. meningitidis encodes

262 a third SPAM, NMB1466/NP_274965, that also falls within Cluster 1A. N. gonorrhoeae TXISS

263 are represented in more nodes than N. meningitidis, notably occupying $19.3 \%$ of nodes in

264 subcluster 1B (Fig. 2). Taken together, these data indicate that Neisseria may be using TXISS to

265 secrete both lipoproteins and soluble products. 
The Type Eleven Secretion System

There is a broadly conserved role for the TXISS in host-microbe interactions. Using the

Steinernema-Xenorhabdus symbiosis we have demonstrated that the composition of TXISS in a

268 bacterial symbiont genome correlates with host organism. During the evolutionary history of

269 Xenorhabdus the gain or loss of TXISS loci correlated with host switching events (Fig. 5). Given

270 the conservation of the TXISS Hrp locus, amongst all Xenorhabdus and throughout human

271 microbiome constituents, it will be important in the future to examine the regulation of Hrp

272 TXISS-dependent secretion and the roles of Hrp machinery in binding and acquiring host metals

273 in a mucosal environment.

274 Finally, the whole family network first generated from the PF04575 (DUF560) dataset

275 included cluster 5, comprising 14 nodes of Klebsiella homologs and 2 nodes with Klebsiella and

276 Escherichia homologs. However, based on its limited number of nodes and predicted topology

277 differences relative the rest of the network (16-stranded vs. 14-stranded barrel), cluster 5 was

278 filtered out prior to our in-depth analysis. Cluster 5 homologs are annotated as PgaA/HmsH

279 OMPs responsible for the synthase-dependent secretion of the exopolysaccharide poly-beta-1,6

280 N-acetyl-D-glucosamine (PNAG), which is necessary for biofilm formation $(47,48)$. The

281 structure of the C-terminus of E. coli PgaA has been solved and forms a 16-stranded porin

282 through which PNAG is exported (49). Although PgaA homologs exist in many bacteria,

283 including $X$. nematophila, the only PgaA homologs represented in any cluster of the original

284 network output were those from Klebsiella spp., further strengthening exclusion of cluster 5 from

285 the TXISS network presented here (48). However, despite the topological and substrate

286 (polysaccharide vs. protein) differences, the PF04575 assignment of cluster 5 members hints that

287 there could be evolutionary or structural parallels between the PNAG exopolysaccharide (EPS)

288 synthase-dependent secretion system and TXISS. PgaA is a component of one of several 
The Type Eleven Secretion System

machineries responsible for export of EPS polymers including PNAG, colonic acid, alginate, cellulose, Psl, and Pel that comprise biofilm matrices of Gram-negative bacteria (47). In each case, like the TXISS system described here, the beta-barrel porin either has or associates with another protein that has TPR domains (47). The TPR region of E. coli PgaA is necessary for PNAG secretion and biofilm formation (49) and the TPR region of PelB modulates the activity

294 of the polymer-modifying enzyme PelA, with impacts the chemistry of the resulting polymer

295 (50). TXISS TPR domains may similarly modulate the activities of other proteins that influence

296 secretion of the TXISS protein substrates. Indeed, X. nematophila expressing versions of the

297 NilB TPR domain with small deletions display colonization defects that are ameliorated by

298 deletion of the entire N-terminal periplasmic domain (11). It will be interesting for future studies

299 to examine the commonalities between the TXISS and EPS synthase-dependent secretion

300 systems and if there is any coordination in secretion of their respective substrates and/or host-

301 associated phenotypes including aggregation and biofilm formation.

302

Materials and Methods

\section{DUF560 sequence similarity network analysis} predicted DUF560-domain-containing protein sequences from Interpro 73 and Uniprot 2019-02

307 (accessed 4.24.19) and BLAST for similarity $(16,17,20)$. Representative networks collapsed

308 nodes which shared $\geq 40 \%$ identity. On an EFI-EST network, edges are drawn according to a

309 database-independent value called alignment score. A greater alignment score requirement

310 means draws fewer edges. For separation of DUF560-domain-containing proteins an alignment

311 score of 38 was chosen (Fig. 1). For subcluster 1A 89 was chosen and for subcluster 1B 100 was 
The Type Eleven Secretion System

312 chosen (Supplemental Fig. 1). The EFI-EST Color SSN tool was used to assign cluster numbers.

313 Networks were visualized and interpreted using Cytoscape v3.7.1 (51) and Gephi v0.9.2 (52).

314 Nodes were arranged with the Fruchterman-Reingold force-directed layout algorithm (53).

315 The contents of each cluster were compared to Pfam DUF560 (PF04575) to ensure that

316 clusters were legitimate DUF560 proteins (21). Any clusters for which fewer than 18\% of

317 sequences were present in Pfam, or which included fewer than 20 sequences were excluded from

318 downstream analysis. This filtering removed cluster 5, which was composed mostly of Klebsiella

319 pneumoniae PgaA. This generated a sequence similarity network that contains 10 clusters, 1222

320 nodes, and 52190 edges with 1589 TaxIDs represented (Fig. 1). Using NCBI Taxonomy

321 Browser, each node was examined and manually curated for the isolates' environmental origin(s)

322 among the following categories: water, soil, plant, mammal, animal, invertebrate, nematode,

323 built (environments such as sewage, bioreactors, etc.), multiple environments, and unclassified

324 (Supplemental Table 1). If no citation was available, the isolation source was searched for in the

325 biosamples or bioprojects records. If neither resource was available, strain source was searched

326 for through other resources (NCBI linkout, Google search). A node was assigned an environment

327 if the majority of strains within the node fell into that environment. If a node had no majority

328 environment, the category "multiple environments" was assigned. Any node with animal

329 associated microbes that did qualify as mammal, insect, or nematode associated was designated

330 as generic "animal associated". For fine scale interpretation, analysis focused on cluster 1 and its

331 subclusters (Fig. 2; Supplemental Fig. 1)

332 Three different techniques were used to determine if DUF560 proteins present within our

333 network were genomically associated with TbpB_B_D-domain-containing proteins, hereafter

334 TbpBBD-domain-containing proteins. First, using EFI-GNN, genome-neighborhood-networks 
The Type Eleven Secretion System

were generated for subcluster 1A (Alignment Score 89; 20 ORFs around), subcluster 1B

(Alignment Score 38; 10 ORFs around), and subcluster 1C (Alignment Score 38; 10 ORFs

Xenorhabdus were manually annotated. All three datasets were combined for a total of 851 non-

341 redundant protein pairs (Supplemental Table 2). SignalP-5 $(54,55)$ was used to predict the signal

342 peptides of all TbpBBD-domain-containing proteins. These predictions were used to annotate

343 each node (Fig. 2C). Any node that was associated with both signal peptide bearing proteins and

344 those with no predicted signal peptide were annotated according to the signal peptide bearing

345 proteins.

347 DUF560 genome neighborhood analysis

Subclusters 1A-C were separated and analyzed in EFI-EST with an alignment score of 38 as described above. Each network was then analyzed through EFI-GNN and visualized in

350 Cytoscape v3.7.1 (51) (Alignment Score 38; 10 ORFs up and downstream).

\section{Phyre $^{2}$ analysis}

354 rettgeri(PROVETT_08181/PROVETT_05852), and Proteus mirabilis (WP_134940027.1) were

355 collected and the first 22 amino acids were trimmed to remove the signal sequences. These

356 sequences were entered into the Phyre $^{2}$ Protein Homology/analogy Recognition Engine v.2.0 to

357 predict potential 3-dimensional structures (25). The top predicted structural model output for all 
The Type Eleven Secretion System

three proteins, hemophilin (6OM5), was used to generate structural models (Supplemental Fig.

2). Subsequent PDB files were visualized with Protean 3D v15. (Protean 3D®. Version 15.0.

DNASTAR. Madison, WI).

\section{Co-expression of HrpB and HrpC}

Primers and strains in Supplemental Table 5. Gene synthesis was performed by

364 Genscript $^{\circledR}$ to synthesize the gene encoding HrpB25_26insDYKDDDDK (henceforth HrpBFLAG). Genscript ${ }^{\circledR}$ subsequently cloned HrpB-FLAG into multiple cloning site 2 (MCS2) of pETDuet-1. The genomic region containing HrpC was amplified from the $X$. nematophila ATCC 19061 (HGB800) genome using primers \#11-12, digested with restriction enzymes SacI and SalI, and ligated into the multicloning site of pUC19. Site directed mutagenesis was used to add a Cterminal FLAG-tag onto HrpC using primers \#13-14. The gene encoding HrpC1_2insV246_247insDYKDDDDK (henceforth HrpC-FLAG) was amplified from pUC19

371 using the primers \#15-16. The gene product was ligated into multiple cloning site 1 (MCS1) of

372 both pETDuet-1 and pETDuet-1/HrpB-FLAG, resulting in the expression plasmids pETDuet-

373 1/HrpC-FLAG and pETDuet-1/HrpC-FLAG/HrpB-FLAG. Correct insertion of all genes was

374 confirmed using primers \#17-20 and Sanger sequencing at the University of Tennessee

375 Genomics Core.

377 (HGB2402 and HGB2459). All strains were grown solely in defined medium with $150 \mathrm{ug} / \mathrm{ml}$ 378 ampicillin as previously described (56). Bacteria producing each expression plasmid were 379 subcultured into $100 \mathrm{ml}$ of broth at an initial OD600 of 0.028 . Cultures were grown for 18 hours 380 at $37^{\circ} \mathrm{C}$ to reach late logarithmic growth and then induced with $500 \mu \mathrm{M}$ IPTG. One hour after 
The Type Eleven Secretion System

381 induction a $700 \mu \mathrm{l}$ sub-sample of each culture was taken and filter sterilized for subsequent

382 protein precipitation. At 2.5 hours the whole cells were separated from the remaining supernatant

383 and lysed open via a bead beater. Remaining supernatants were filter sterilized. Whole cell

384 lysates were equalized via Bradford assay. For 1 and 2.5 hour supernatant samples $700 \mu 1$ of

385 supernatant was precipitated via 10\% Trichloroacetic acid incubation (57).

386 Samples were run on 1\% SDS-PAGE gels. For lysates, wells were loaded with 9.5ug of

387 protein. For supernatants, wells were loaded with the precipitate of $350 \mu 1$ of media. Western

388 blots were probed with a rat derived $\alpha$-FLAG tag primary antibody and an $\alpha$-IgG secondary

389 antibody that fluoresces at $680 \mathrm{~nm}$. Intensities for FLAG reactive bands were recorded. A

390 distinct band from the Coomassie stained gel was used as a loading control to normalize

391 intensities of supernatant samples prior to analysis (Fig. 4, Supplemental Fig. 3). For whole cell

392 samples the data were analyzed with an unpaired t-test. For supernatant samples a two-way

393 ANOVA was performed alongside Sidak's multiple comparisons test.

\section{Phylogenetic tree generation}

Phylogenetic analysis was performed as described previously (58). Briefly, select

397 Xenorhabdus and Photorhabdus species were analyzed using MicroScope MaGe’s Gene

398 Phyloprofile tool $(34,39)$ to identify homologous protein sets which were conserved across all

399 assayed genomes. Putative paralogs were excluded from downstream analysis to ensure homolog

400 relatedness, resulting in 665 homologous sets (Supplemental Table 5). Homolog sets were

401 retrieved via locus tag indexing using BioPython (59), individually aligned using Muscle

402 v3.8.31(60), concatenated using Sequence Matrix v1.8 (61), and trimmed of nucleotide gaps 
The Type Eleven Secretion System

using TrimAL v1.3 (62). JmodelTest v2.1.10 (63) was used to choose the GTR+ $\gamma$ substitution

404

405

406

407

408

409

410

411

412

413

414

415

416

417

418

419

420

421

422

423

424

425

model for maximum likelihood and Bayesian analysis.

For nematode phylogenetic analysis select Steinernema and Heterorhabditis species were analyzed. The Internal Transcribed Spacer, 18S rRNA, 28S rRNA, Cytochrome Oxidase I, and 12S rRNA loci were collected from Genbank's as available and used as homologous sets (Supplemental Table 4). Nematode species which had fewer than 3 of the 5 loci sequenced were excluded from downstream analysis. Homologous sets were individually aligned, concatenated, and trimmed using the same methods as the Xenorhabdus sequences. JmodelTest v2.1.10 (63) was used to choose the GTR $+\gamma+$ I substitution model.

Maximum likelihood analyses were performed via RAxML v8.2.10 (64) using rapid bootstrapping and 1000 replicates, and were visualized via Dendroscope v3.6.2 (65). Nodes with less than $60 \%$ bootstrap support were collapsed. Bayesian analyses were performed via MrBayes v3.2.6 with BEAGLE (66-68) on the Cipres Science Gateway platform (69). 500,000 MCMC replicates were performed for the bacterial tree, 4,000,000 were performed for the nematode tree. $25 \%$ of replicates were discarded as burn-in, and posterior probabilities were sampled every 500 replicates. Two runs were performed with 3 heated and one cold chain. The final standard deviation of split frequencies was 0.000000 for the bacterial tree, and 0.002557 for the nematode tree. Bayesian trees were visualized with FigTree v1.4.4 (70). Bayesian and maximum likelihood methods generated phylogenies with consistent topologies (Fig. 5; Supplemental Fig. 5).

\section{WT vs. Anil colonization of $S$. anatoliense, $S$. carpocapsae, and $S$. websteri}

Strains are described in Supplemental Table 5. Bacteria free eggs of S. anatoliense, $S$.

carpocapsae, and $S$. websteri were generated (41) and exposed to a Nil locus mutant 
The Type Eleven Secretion System

426 (HGB1495), and a Nil locus complemented strain (HGB1496) grown on lipid agar plates for 2

427 days at $25^{\circ} \mathrm{C}(11)$. Lipid agar plates were placed into White traps 1 week after plating eggs to

428 collect infective juvenile (IJ) nematodes. Nematode colonization was visualized using

429 fluorescence microscopy on the Keyence BZX-700 to observe GFP expressing bacteria in the

430 receptacle. This was done for each species in biological triplicate and technical duplicate. To

431 determine the number of CFU per IJ, nematodes were surface sterilized, and ground for 2 min

432 using a Fisher brand motorized tissue grinder (CAT\# 12-1413-61) to homogenize the nematodes

433 and release colonizing bacteria. Serial dilutions in PBS were performed and plated on LB agar,

434 which were incubated at $30^{\circ} \mathrm{C}$ for 1 day before enumerating CFUs (Fig. 6). To calculate the CFU

435 per colonized IJ, the percent colonized nematodes was divided by the CFU/IJ for each biological

436 replicate. The data were analyzed using a one-way ANOVA with Tukey's multiple comparison's

437 test to compare the mean of each treatment.

438

439 Acknowledgements:

440 This work was supported by a grant from the National Science Foundation (IOS-

441 1353674) to HGB and KTF and the University of Tennessee-Knoxville to HGB. TJM was

442 supported by an NIH National Research Service Award T32-GM07215.

443

444 Author Contributions:

445 TJM, ASG, and HGB wrote the text of this article and composed the figures.

446 Bioinformatic analysis was performed by ASG and TJM. Phylogenetic analysis was performed

447 by ASG. Cloning and experiments were performed by ASG and TJM. KTF provided sustained

448 intellectual contributions to the research design and edits to the text and figures. 
The Type Eleven Secretion System

Additional Information:

The authors have no competing interests that might be perceived to influence the results

and/or discussion reported in this paper. A previous version of this article was published on

should be addressed to H. Goodrich-Blair at hgblair@utk.edu

\section{References:}

1. Hooda Y, Lai CC, Judd A, Buckwalter CM, Shin HE, Gray-Owen SD, Moraes TF. 2016. Slam is an outer membrane protein that is required for the surface display of lipidated virulence factors in Neisseria. Nat Microbiol 1:16009. host; a bird's eye view. Biometals 23:601-11. regulation, structure, and function. Annual Review of Microbiology 64:43-60.

473 7. da Silva RAG, Karlyshev AV, Oldfield NJ, Wooldridge KG, Bayliss CD, Ryan A, 474 Griffin R. 2019. Variant Signal Peptides of Vaccine Antigen, FHbp, Impair Processing Affecting 475 Surface Localization and Antibody-Mediated Killing in Most Meningococcal Isolates. Front 476 Microbiol 10:2847.

477 8. Akhurst RJ. 1983. Neoplectana species: specificity of association with bacteria of the 478 genus Xenorhabdus. Exp Parasitol 55:258-263.

479 9. Chaston JM, Suen G, Tucker SL, Andersen AW, Bhasin A, Bode E, Bode HB, 480 Brachmann AO, Cowles CE, Cowles KN, Darby C, de Leon L, Drace K, Du Z, Givaudan A, 
The Type Eleven Secretion System

Herbert Tran EE, Jewell KA, Knack JJ, Krasomil-Osterfeld KC, Kukor R, Lanois A, Latreille P, Leimgruber NK, Lipke CM, Liu R, Lu X, Martens EC, Marri PR, Medigue C, Menard ML, Miller NM, Morales-Soto N, Norton S, Ogier JC, Orchard SS, Park D, Park Y, Qurollo BA, Sugar DR, Richards GR, Rouy Z, Slominski B, Slominski K, Snyder H, Tjaden BC, van der Hoeven R, Welch RD, Wheeler C, Xiang B, Barbazuk B, et al. 2011. The entomopathogenic bacterial endosymbionts Xenorhabdus and Photorhabdus: convergent lifestyles from divergent genomes. PLoS One 6:e27909.

10. Heungens K, Cowles CE, Goodrich-Blair H. 2002. Identification of Xenorhabdus nematophila genes required for mutualistic colonization of Steinernema carpocapsae nematodes. Mol Microbiol 45:1337-53.

11. Bhasin A, Chaston JM, Goodrich-Blair H. 2012. Mutational analyses reveal overall topology and functional regions of NilB, a bacterial outer membrane protein required for host association in a model of animal-microbe mutualism. J Bacteriol 194:1763-76.

12. Blatch GL, Lassle M. 1999. The tetratricopeptide repeat: a structural motif mediating protein-protein interactions. Bioessays 21:932-9.

13. Cowles CE, Goodrich-Blair H. 2004. Characterization of a lipoprotein, NilC, required by Xenorhabdus nematophila for mutualism with its nematode host. Mol Microbiol 54:464-77.

14. Cowles CE, Goodrich-Blair H. 2008. The Xenorhabdus nematophila nilABC genes confer the ability of Xenorhabdus spp. to colonize Steinernema carpocapsae nematodes. J Bacteriol 190:4121-8.

15. d'Enfert C, Chapon C, Pugsley AP. 1987. Export and secretion of the lipoprotein pullulanase by Klebsiella pneumoniae. Mol Microbiol 1:107-16.

16. Gerlt JA, Bouvier, J.T., Davidson, D.B., Imker, H.J., Sadkhin, B., Slater, D.R., Whalen, K.L. 2015. Enzyme Function Initiative-Enzyme Similarity Tool (EFI-EST): A web tool for generating protein sequence similarity networks. Biochemica et Biophysica Acta 1854:10191037.

17. Zallot R, Oberg, N.O., Gerlt, J.A. 2018. 'Democratized'genomicenzymologywebtoolsforfunctionalassignment. Current Opinion in Chemical Biology 47:77-85.

18. Zallot R, Oberg N, Gerlt JA. 2019. The EFI Web Resource for Genomic Enzymology Tools: Leveraging Protein, Genome, and Metagenome Databases to Discover Novel Enzymes and Metabolic Pathways. Biochemistry 58:4169-4182.

19. Ostberg KL, DeRocco, A.J., Mistry, S.D., Dickinson, M.K., Cornelissen, C.N. 2013. Conserved Regions of Gonococcal TbpB Are Critical for Surface Exposure and Transferrin Iron Utilization. Infection and Immunity 81:3442-3450. 
The Type Eleven Secretion System

20. Gerlt JA. 2017. Genomic Enzymology: Web Tools for Leveraging Protein Family Sequence-Function Space and Genome Context to Discover Novel Functions. Biochemistry 56:4293-4308.

21. El-Gebali S, Mistry J, Bateman A, Eddy SR, Luciani A, Potter SC, Qureshi M, Richardson LJ, Salazar GA, Smart A, Sonnhammer ELL, Hirsh L, Paladin L, Piovesan D, Tosatto SCE, Finn RD. 2019. The Pfam protein families database in 2019. Nucleic Acids Research 47:D427-D432.

22. Choe K, Mitchell Laboratory. 2018. RODEO (Rapid ORF Description \& Evaluation Online) is an algorithm to help biosynthetic gene cluster (BGC) analysis, with an emphasis on ribosomal natural product (RiPP) discovery., $\mathrm{p}$ http://ripp.rodeo/advanced.html, University of Illinois at Urbana-Champaign.

23. Latham RD, Torrado M, Atto B, Walshe JL, Wilson R, Guss JM, Mackay JP, Tristram S, Gell DA. 2020. A heme-binding protein produced by Haemophilus haemolyticus inhibits nontypeable Haemophilus influenzae. Mol Microbiol 113:381-398.

24. Kelley LA, Sternberg MJ. 2009. Protein structure prediction on the Web: a case study using the Phyre server. Nat Protoc 4:363-71.

25. Kelley LA, Mezulis S, Yates CM, Wass MN, Sternberg MJ. 2015. The Phyre2 web portal for protein modeling, prediction and analysis. Nat Protoc 10:845-58.

26. Einhauer A, Jungbauer A. 2001. The FLAG peptide, a versatile fusion tag for the purification of recombinant proteins. J Biochem Biophys Methods 49:455-65.

27. Fantappie L, Irene C, De Santis M, Armini A, Gagliardi A, Tomasi M, Parri M, Cafardi V, Bonomi S, Ganfini L, Zerbini F, Zanella I, Carnemolla C, Bini L, Grandi A, Grandi G. 2017. Some Gram-negative lipoproteins keep their surface topology when transplanted from one species to another and deliver foreign polypeptides to the bacterial surface. Mol Cell Proteomics $16: 1348-1364$.

28. Konar M, Rossi R, Walter H, Pajon R, Beernink PT. 2015. A mutant library approach to identify improved meningococcal factor $\mathrm{H}$ binding protein vaccine antigens. PLoS One 10:e0128185.

29. Jackson LA, Ducey TF, Day MW, Zaitshik JB, Orvis J, Dyer DW. 2010. Transcriptional and functional analysis of the Neisseria gonorrhoeae Fur regulon. J Bacteriol 192:77-85.

30. Quillin SJ, Hockenberry AJ, Jewett MC, Seiferta HS. 2018. Neisseria gonorrhoeae exposed to sublethal levels of hydrogen peroxide mounts a complex transcriptional tesponse. mSystems 3:e00156-18.

31. Stohl EA, Criss AK, Seifert HS. 2005. The transcriptome response of Neisseria gonorrhoeae to hydrogen peroxide reveals genes with previously uncharacterized roles in oxidative damage protection. Mol Microbiol 58:520-532. 
The Type Eleven Secretion System

32. Dent AT, Mouriño S, Huang W, Wilks A. 2018. Post-transcriptional regulation of the Pseudomonas aeruginosa heme assimilation system (Has) fine-tunes extracellular heme sensing. J Biol Chem 294:2771-2785.

33. Yukl ET, Jepkorir G, Alontaga AY, Pautsch L, Rodriguez JC, Rivera M, Moënne-Loccoz P. 2010. Kinetic and spectroscopic studies of hemin acquisition in the hemophore HasAp from Pseudomonas aeruginosa. Biochemistry 49:6646-6654.

34. Chaston JM, Murfin KE, Heath-Heckman EA, Goodrich-Blair H. 2013. Previously unrecognized stages of species-specific colonization in the mutualism between Xenorhabdus bacteria and Steinernema nematodes. Cellular Microbiology 15:1545-1559.

35. Kampfer P, Tobias NJ, Ke LP, Bode HB, Glaeser SP. 2017. Xenorhabdus thuongxuanensis sp. nov. and Xenorhabdus eapokensis sp. nov., isolated from Steinernema species. International Journal of Systematic and Evolutionary Microbiology 67:1107-1114.

36. Lee MM, Stock SP. 2010. A multigene approach for assessing evolutionary relationships of Xenorhabdus spp. (gamma-Proteobacteria), the bacterial symbionts of entomopathogenic Steinernema nematodes. J Invertebr Pathol 104:67-74.

37. Snyder HA, Stock SP, Kim SK, Flores-Lara Y, Forst S. 2007. New insights into the colonization and release process of Xenorhabdus nematophila and the morphology and ultrastructure of the bacterial receptacle of its nematode host, Steinernema carpocapsae. Applied and Environmental Microbiology 73:5338-5346.

38. Spiridonov SE, Reid AP, Podrucka K, Subbotin SA, Moens M. 2004. Phylogenetic relationships within the genus Steinernema (Nematoda: Rhabditida) as inferred from analyses of sequences of the ITS1-5.8S-ITS2 region of rDNA and morphological features. Nematology 6:547-566.

39. Vallenet D, Calteau A, Cruveiller S, Gachet M, Lajus A, Josso A, Mercier J, Renaux A, Rollin J, Rouy Z, Roche D, Scarpelli C, Medigue C. 2017. MicroScope in 2017: an expanding and evolving integrated resource for community expertise of microbial genomes. Nucleic Acids Research 45:D517-D528.

40. McGinnis S, Madden TL. 2004. BLAST: at the core of a powerful and diverse set of sequence analysis tools. Nucleic Acids Res 32:W20-5.

41. Murfin KE, Chaston J, Goodrich-Blair H. 2012. Visualizing bacteria in nematodes using fluorescence microscopy. J Vis Exp 68:e4298.

42. Costa TR, Felisberto-Rodrigues C, Meir A, Prevost MS, Redzej A, Trokter M, Waksman G. 2015. Secretion systems in Gram-negative bacteria: structural and mechanistic insights. Nat Rev Microbiol 13:343-59.

43. Zielke RA, Simmons RS, Park BR, Nonogaki M, Emerson S, Sikora AE. 2014. The type II secretion pathway in Vibrio cholerae is characterized by growth phase-dependent expression of exoprotein genes and is positively regulated by sigmaE. Infect Immun 82:2788-801. 
The Type Eleven Secretion System

44. Bendtsen JD, Kiemer L, Fausboll A, Brunak S. 2005. Non-classical protein secretion in bacteria. BMC Microbiol 5:58.

45. Palmer T, Finney AJ, Saha CK, Atkinson GC, Sargent F. 2020. A holin/peptidoglycan hydrolase-dependent protein secretion system. Mol Microbiol doi:10.1111/mmi.14599.

46. Cohen J, Powderly W, Opal S. 2017. Preface to the Fourth Edition, Infectious Diseases, 4th edition. Elsevier.

47. Low KE, Howell PL. 2018. Gram-negative synthase-dependent exopolysaccharide biosynthetic machines. Curr Opin Struct Biol 53:32-44.

48. Whitney JC, Howell PL. 2013. Synthase-dependent exopolysaccharide secretion in Gram-negative bacteria. Trends Microbiol 21:63-72.

49. Wang Y, Andole Pannuri A, Ni D, Zhou H, Cao X, Lu X, Romeo T, Huang Y. 2016. Structural Basis for Translocation of a Biofilm-supporting Exopolysaccharide across the Bacterial Outer Membrane. J Biol Chem 291:10046-57.

50. Marmont LS, Whitfield GB, Rich JD, Yip P, Giesbrecht LB, Stremick CA, Whitney JC, Parsek MR, Harrison JJ, Howell PL. 2017. PelA and PelB proteins form a modification and secretion complex essential for Pel polysaccharide-dependent biofilm formation in Pseudomonas aeruginosa. J Biol Chem 292:19411-19422.

51. Shannon P, Markiel A, Ozier O, Baliga NS, Wang JT, Ramage D, Amin N, Schwikowski B, Ideker T. 2003. Cytoscape: a software environment for integrated models of biomolecular interaction networks. Genome Res 13:2498-504.

52. Bastian M, Heymann S, Jacomy M. 2009. Gephi : An Open Source Software for Exploring and Manipulating Networks. International AAAI Conference on Weblogs and Social Media.

53. Fruchterman TMJ, Reingold EM. 1991. Graph Drawing by Force-directed Placement. Software - Practice and Experience 21:1129-1164.

54. Almagro Armenteros JJ, Tsirigos KD, Sonderby CK, Petersen TN, Winther O, Brunak S, von Heijne G, Nielsen H. 2019. SignalP 5.0 improves signal peptide predictions using deep neural networks. Nat Biotechnol 37:420-423.

55. Nielsen H, Engelbrecht J, Brunak S, von Heijne G. 1997. Identification of prokaryotic and eukaryotic signal peptides and prediction of their cleavage sites. Protein Engineering 10:1-6.

56. Orchard SS, Goodrich-Blair H. 2004. Identification and functional characterization of a Xenorhabdus nematophila oligopeptide permease. Appl Environ Microbiol 70:5621-7.

57. Koontz L. 2014. TCA Precipitation, p 3-10. In Lorsch J (ed), Laboratory Methods in Enzymology: Protein Part C, vol 536. Academic Press Elsevier. 
The Type Eleven Secretion System

58. Murfin KE, Lee MM, Klassen JL, McDonald BR, Larget B, Forst S, Stock SP, Currie CR, Goodrich-Blair H. 2015. Xenorhabdus bovienii strain diversity impacts coevolution and symbiotic maintenance with Steinernema spp. nematode hosts. MBio 6:e00076.

59. Cock PJ, Antao T, Chang JT, Chapman BA, Cox CJ, Dalke A, Friedberg I, Hamelryck T, Kauff F, Wilczynski B, de Hoon MJ. 2009. Biopython: freely available Python tools for computational molecular biology and bioinformatics. Bioinformatics 25:1422-3.

60. Edgar RC. 2004. MUSCLE: multiple sequence alignment with high accuracy and high throughput. Nucleic Acids Res 32:1792-7.

61. Vaidya G, Lohman, D. J., Meier, R. 2010. SequenceMatrix: Concatenation software for the fast assembly of multi-gene datasets with character set and codon information. Cladistics 27:171-180.

62. Capella-Gutiérrez S, Silla-Martínez, J. M., Gabaldón, T. 2009. trimAl: a tool for automated alignment trimming in large-scale phylogenetic analyses. Bioinformatics 25:19721973.

63. Darriba D, Taboada, G. L., Doallo, R., Posada, D. 2012. jModelTest 2: more models, new heuristics and highperformance computing. Nat Methods 9:772.

64. Stamatakis A. 2014. RAxML version 8: a tool for phylogenetic analysis and post-analysis of large phylogenies. Bioinformatics 30:1312-1313.

65. Huson DH, Scornavacca, C. 2012. Dendroscope 3: An Interactive Tool for Rooted Phylogenetic Trees and Networks. Syst Biol 61:1061-1067.

66. Altekar G, Dwarkadas, S., Huelsenbeck, J. P., Ronquist, F. 2004. Parallel Metropolis coupled Markov chain Monte Carlo for Bayesian phylogenetic inference Bioinformatics 20:407415.

67. Ayres DL, Darling, A., Zwickl, D. J., Beerli, P., Holder, M. T., Lewis, P. O., Huelsenbeck, J. O., Ronquist, F., Swofford, D. L., Cummings, M. P., Rambaut, A., Suchard, M. A. 2011. BEAGLE: An Application Programming Interface and High-Performance Computing Library for Statistical Phylogenetics. Syst Biol 61:170-173.

68. Ronquist F, Teslenko, M., van der Mark, P., Ayres, D. L., Darling, A., Hohna, S., Larget, B., Liu, L., Suchard, M. A., Huelsenbeck, J. P. 2012. MrBayes 3.2: Efficient Bayesian Phylogenetic Inference and Model Choice Across a Large Model Space. Syst Biol 61:539-542.

69. Miller MA, Pfeiffer, W., Schwartz, T. Creating the CIPRES Science Gateway for inference of large phylogenetic trees, p 1-8. In (ed), Proceedings of the Gateway Computing Environments Workshop (GCE),

70. Rambaut A. 2018. FigTree v1.4.4 phttp://tree.bio.ed.ac.uk/software/figtree/. 
bioRxiv preprint doi: https://doi.org/10.1101/2020.01.20.912956; this version posted February 19, 2021. The copyright holder for this preprint (which was not certified by peer review) is the author/funder, who has granted bioRxiv a license to display the preprint in perpetuity. It is made available under aCC-BY-NC-ND 4.0 International license.

The Type Eleven Secretion System

\section{Supplemental Data}

\begin{tabular}{|c|c|c|}
\hline & $\begin{array}{c}\text { Subcluster } 1 \mathrm{~A} \\
\text { Alignment Score 89; Rep. Nodes } 40 \%\end{array}$ & $\begin{array}{c}\text { Subcluster 1B } \\
\text { Alignment Score 100; Rep. Nodes } 40 \%\end{array}$ \\
\hline $\begin{array}{l}\text { Taxonomic } \\
\text { classification } \\
\text { (Family) } \\
\text { Neisseriaceae } \\
\text { Moraxellaceae } \\
\text { Enterobacteriaceae } \\
\text { Pasteurellaceae } \\
\text { Morganellaceae } \\
\text { Xanthomonadaceae } \\
\text { Oceanospirillaceae } \\
\text { Pseudomonadaceae } \\
\text { Comamonadaceae } \\
\text { Alcaligenaceae } \\
\text { Vibrionaceae } \\
\text { Multiple/Other } \\
\text { NA }\end{array}$ & NilB Cluster & 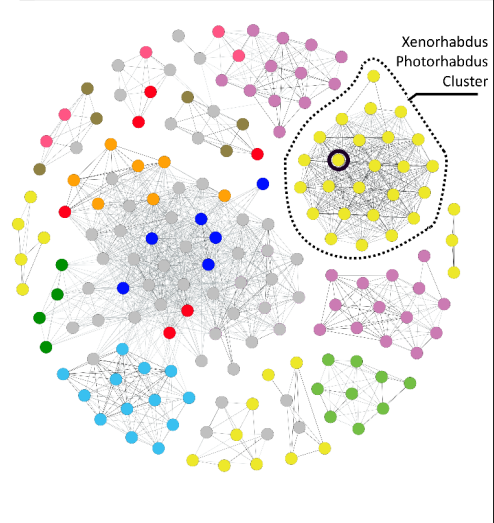 \\
\hline $\begin{array}{l}\text { TbpBBD } \\
\text { assocation } \\
\text { TbpBBD sol } \\
\text { co-occurrence } \\
\text { TbpBBD ip } \\
\text { co-occurrence } \\
\text { TbpBBDsond TbpBBD }{ }_{\text {iip }} \\
\text { co-occurrence } \\
\text { TbpBBD without } \\
\text { signal peptide } \\
\text { No detected TbpBBD } \\
\text { co-occurrence }\end{array}$ & Cluster & 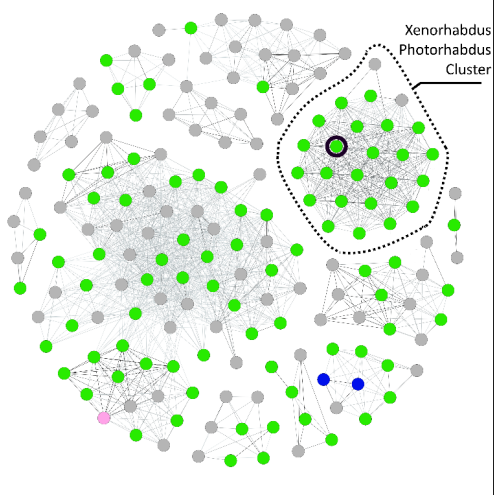 \\
\hline $\begin{array}{l}\text { Characterized } \\
\text { proteins }\end{array}$ & pB assoc & AA associated/Slam2 \\
\hline
\end{tabular}

Supplemental Figure 1. Increased stringency separates subcluster 1A and 1B into

661 functional groups. A series of stringent EFI-EST sequence similarity networks highlights detail

662 in cluster 1 of the DUF560 homologs. Edge darkness demonstrates similarity. Node positioning

663 was optimized using the Fruchterman-Reingold algorithm (53). Dotted lines indicate

664 hypothetical functional clusters based on previous molecular data. Circled nodes indicate

665 proteins which have been molecularly characterized. Subclusters 1A and 1B were analyzed

666 separately to allow fine tuning of alignment score (89 and 100 respectively). Networks were

667 color coded to display either taxonomic categories or co-inheritance with TbpBBD-domain

668 containing proteins. 
The Type Eleven Secretion System

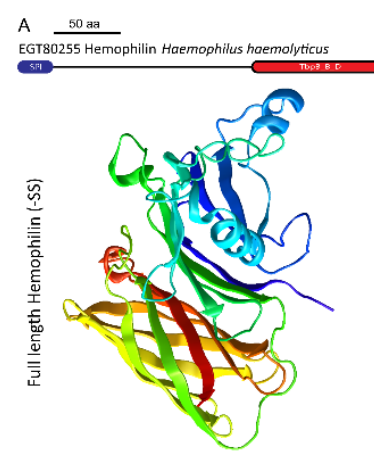

B

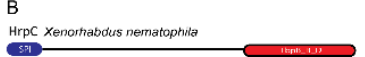

C

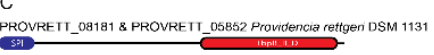

D

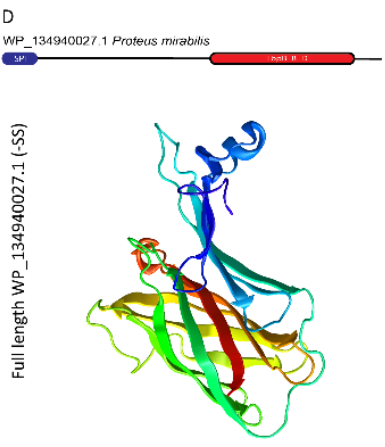

670 Supplemental Figure 2. Phyre ${ }^{2}$ models of select TbpBBD sol $_{\text {proteins. }}$ TbpBBD ${ }_{\text {sol }}$ proteins,

671 lacking the signal sequence (-SS), from X. nematophila (HrpC), P. rettgeri (PROVRETT_08181

672 and 05852), and P. mirabilis (WP_134940027.1) were queried through the Phyre ${ }^{2}$ Protein

673 Homology/analogy Recognition Engine v. 2.0

674 (http://www.sbg.bio.ic.ac.uk/phyre2/html/page.cgi?id=index) (25). The top predicted structural

675 model output for each is shown alongside the solved crystal structure of hemophilin from $H$.

676 haemolyticus (protein data bank file 6OM5) which the algorithm selected as the template for all

677 queries (23). 

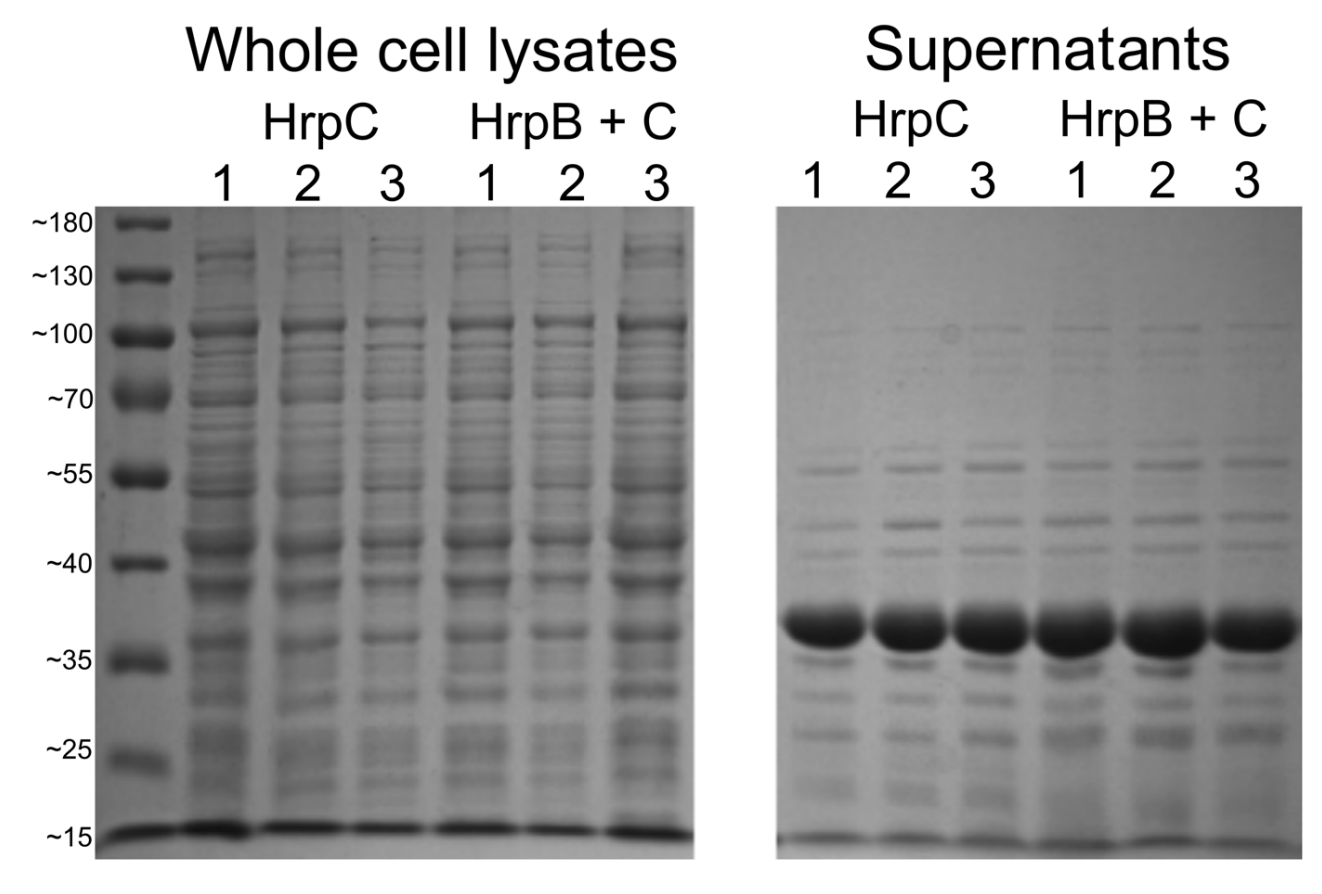

679 Supplemental Figure 3. Supernatant samples have distinct protein profile. Coomassie

680 stained SDS-PAGE gels showing side by side comparison of protein banding in whole cell

681 lysates and supernatant samples of $E$. coli expressing the indicated proteins. Units on the left side

682 are in kilodaltons. Expression of HrpB does not cause cell lysis or nonspecific protein export, if

683 it did the banding pattern of the supernatant would look more like that of the lysates. The

684 increase in supernatant $\mathrm{HrpC}$ is faintly visible at $\sim 27$ kilodaltons in the last three supernatant

685 sample lanes. All experiments were repeated in biological triplicate as labeled above the lanes. 
The Type Eleven Secretion System

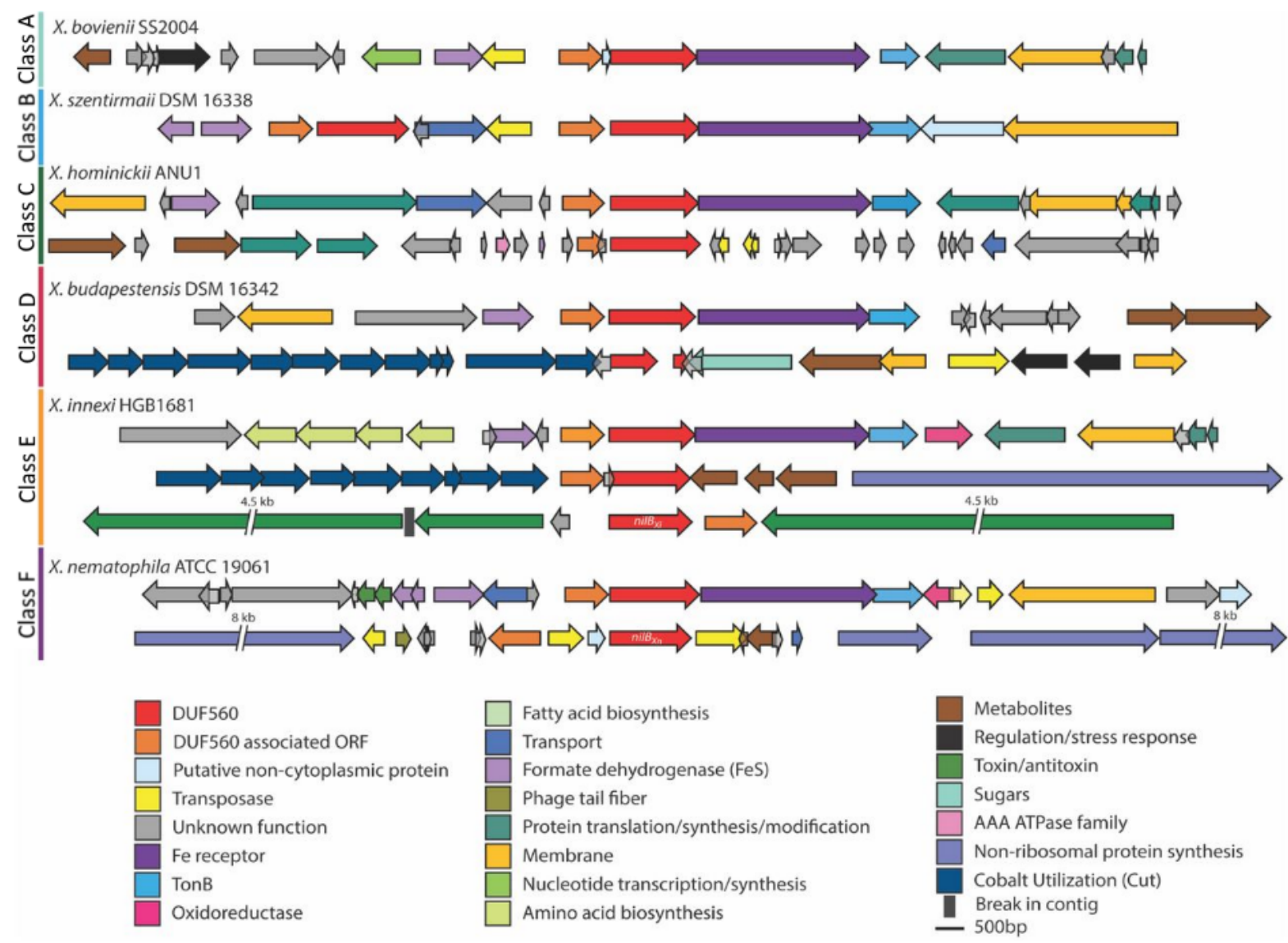

Supplemental Figure 4. Representative genomes of Xenorhabdus DUF560 Classes A-F.

Schematic diagrams of Xenorhabdus TXISS loci representing each of the six classes defined in the text. One species from each of the classes was selected for presentation. Box arrows represent open reading frames (ORFs), which are color coded according to predicted annotated

691 function as indicated by the legend. The DUF560 homolog is shown in red and the predicted of the gap is indicated above the break line shown within such ORFs. 
The Type Eleven Secretion System
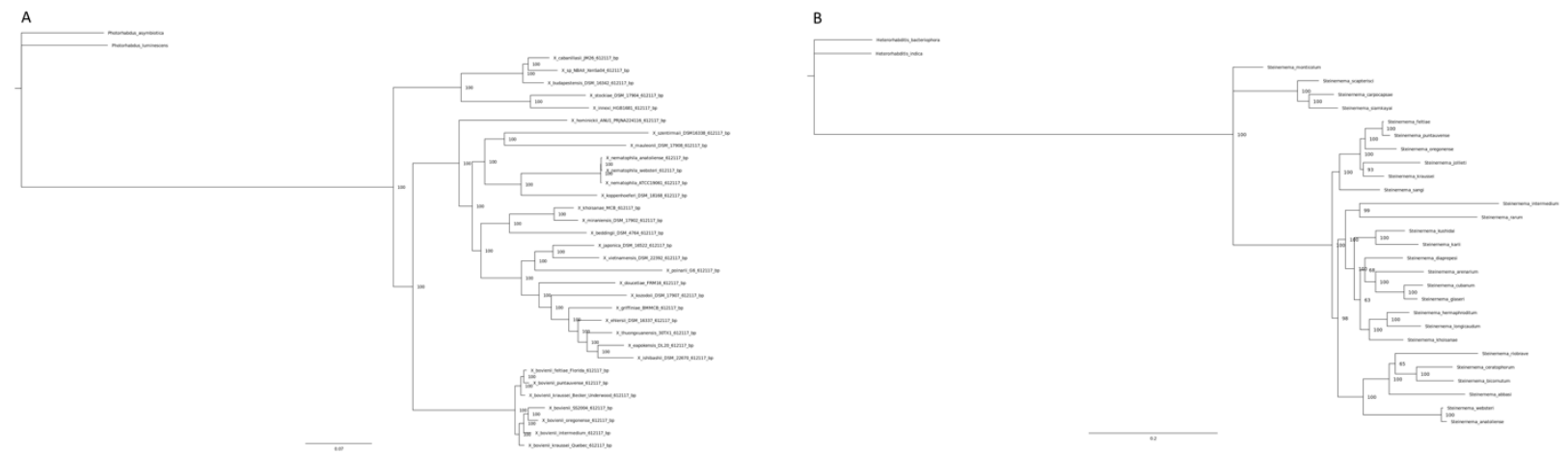

699 Supplemental Figure 5. Bayesian posterior probability phylogenies. A) Phylogram of select

700 Xenorhabdus bacteria, based on concatenations of 665 conserved core genes. Numbers indicate

701 posterior probability values. Distances indicate substitutions per base pair. B) Bayesian

702 phylogeny of select entomopathogenic nematodes, based on concatenations of the ITS, 18S

703 rRNA, 28S rRNA, COI, and 12S rRNA loci. Two members of the sister taxon Photorhabdus

704 were chosen as an outgroup. Loci are recorded in Supplemental table 4. 
The Type Eleven Secretion System

705 Supplemental Table 1. DUF560 sequence similarity network clusters 1-4 and 6-11 node

706 table, with subcluster, environmental annotations, and sources (when possible)

707 Associated file: TableS1_Clusters1-4,6-11WithEnvTax.xlsx

708 Supplemental Table 2. DUF560 TbpBBD protein Co-occurrence

709 Associated file: TableS2_DUF560TbpBBDCo-occurrence.xlsx

710 Supplemental Table 3. Xenorhabdus DUF560 classes

711 Associated file: TableS3_XenorhabdusDUF560Classes.xlsx

712 Supplemental Table 4. Loci tags used in phylogenetic analysis of Xenorhabdus and

713 Steinernema species

714 Associated file: TableS4_CombinedLociTable

715 Supplemental Table 5. Primer and strain list

716 Associated file: TableS5_Primers_AndStrains.xlsx 